\title{
18F-PSMA-1007 PET/CT in Primary Prostate Cancer: Using tPSA to Detect Distant Metastasis and Incorporating SUVmax to Characterize Primary Prostate Cancer and Distant Metastasis
}

\section{Zhuonan Wang}

The First Affiliated Hospital of Xi'an Jiaotong University

Anqi Zheng

The First Affiliated Hospital of Xi'an Jiaotong University

Weixuan Dong

The First Affiliated Hospital of Xi'an Jiaotong University

Xiang Liu

The First Affiliated Hospital of Xi'an Jiaotong University

\section{Wang Yuan}

The First Affiliated Hospital of Xi'an Jiaotong University

\section{Fan Gao}

The First Affiliated Hospital of Xi'an Jiaotong University

Xiaoyi Duan ( $\nabla$ duanxy@mail.xjtu.edu.cn )

The First Affiliated Hospital of Xi'an Jiaotong University https://orcid.org/0000-0003-4595-9410

\section{Research Article}

Keywords: Primary Prostate Cancer, 18F-PSMA-1007, PET/CT, SUVmax, tPSA level, Distant Metastasis

Posted Date: February 16th, 2021

DOl: https://doi.org/10.21203/rs.3.rs-201417/v1

License: (c) (1) This work is licensed under a Creative Commons Attribution 4.0 International License. Read Full License 


\section{Abstract}

\section{Purpose}

To evaluate the diagnostic performance of total prostate-specific antigen (tPSA) for primary prostate cancer (PCa) distant metastasis in ${ }^{18} \mathrm{~F}-\mathrm{PSMA}-1007 \mathrm{PET} / \mathrm{CT}$, and the potential mediation role of tPSA level on the maximum standardized uptake value (SUVmax) of different metastasis stages.

\section{Methods}

Retrospective analysis was performed on 101 patients admitted consecutively between March 2019 and June 2020 with biopsy proven PCa who were referred for PET/CT. The diagnostic performance of tPSA to detect distant metastasis was evaluated using receiver operating characteristic curve (ROC) and logistic regression analyses. SUVmax was compared across different metastatic stages with either low or high tPSA level using analysis of variance (ANOVA). A path analysis was conducted to evaluate the mediating effect of tPSA level on the relation between SUVmax of primary PCa and SUVmax of metastatic foci.

Results

At a value of 29.01 , the prediction tPSA demonstrated a sensitivity of $57.14 \%$ and a specificity of $73.68 \%$ for detecting distant metastasis with an area under the curve of $0.656(P=0.004)$. The prediction tPSA level was the only significant predictive variable in a logistic regression model $(P=0.008$, odds ratio $=$ 3.273). SUVmax was significantly different between primary prostate tissue with metastasis and metastasis foci only when tPSA $\geq 29.01$ ( $P=0.001)$. In addition, for those with metastasis foci, SUVmax was significantly higher in those with tPSA $\geq 29.01$ than those with tPSA $<29.01(P=0.034)$. Both tPSA and SUVmax associated with risk of metastasis $(P=0.008, P=0.014)$, and tPSA was found to have a partial mediating effect on SUVmax of primary PCa and metastases foci $(P<0.05)$.

Conclusion

A prediction tPSA level of 29.01 can help predict the distant metastases in primary PCa. The incorporation of ${ }^{18} \mathrm{~F}-\mathrm{PSMA}-1007 \mathrm{PET} / \mathrm{CT}$ in $\mathrm{PCa}$ evaluation may better characterize the metastasis lesions.

\section{Introduction}

Prostate cancer $(\mathrm{PCa})$ is one of the most common malignant tumor and leading causes of mortality for men worldwide $[1,2]$. The biological behaviors of PCa malignancy are largely heterogeneous and have direct impacts on prognostic grouping and treatment planning. Therefore, an accurate systemic staging of PCa before treatment is crucial. Assessments of the distant metastatic status for high-risk PCa patients have recently received increasing attention due to the heightening mortality rate $[3,4]$. Total prostate-specific antigen (tPSA) is the most widely used biomarker when screening for primary PCa. According to the American Urological Association (AUA) and the European Association of Urology (EAU) 
guidelines, patients with tPSA $>20 \mathrm{ng} / \mathrm{mL}$ are recommended to undergo bone scintigraphy imaging to detect distant metastases [5-7]. However, the utility of tPSA is limited due to its organ but not cancerspecific nature and a lack of gold standard measurement procedure $[8,9]$. The conventional methods for detecting distant metastases are computed tomography or bone scintigraphy scan, which depend largely on morphological information and can detect only larger lesions [10]. The overall lack of sensitive methods to detect primary PCa distant metastases results in an overuse of biopsy and repeated imaging scans, which is associated with higher costs and risks for adverse effects such as infection and bleeding. It is, therefore, crucial to identify other objective and accurate imaging biomarkers for detecting primary PCa and metastatic lesions.

PSMA is a type II transmembrane protein, and its expression level is positively correlated with the degree of malignant tumors. The ability of PSMA to easily penetrate tissues and diffuse with solid tumor lesions can reflect the statuses of metastasis [11-13]. In recent years, PET/CT techniques targeting PSMA have gained increasing popularity in clinical practice. ${ }^{18} \mathrm{~F}$-PSMA-1007 was identified as a reliable PSMAtargeting PET-tracer. $[14,15] .{ }^{18} \mathrm{~F}$-labeled agents enable large-scale radiosynthesis and allowing for a higher number of patient studies with a longer physical half-life. Due to its higher spatial resolution images and non-urinary excretion that reduces urinary clearance, ${ }^{18} \mathrm{~F}-\mathrm{PSMA}-1007 \mathrm{PET} / \mathrm{CT}$ bears a great potential to facilitate the detection of primary PCa and metastatic lesions $[16,14,17]$. Prior studies have established the association between PSMA uptake in primary PCa and pre-treatment PSA levels $[18,19]$. It is, therefore, believed that ${ }^{18} \mathrm{~F}-\mathrm{PSMA}-1007 \mathrm{PET} / \mathrm{CT}$ can be used as an imaging biomarker to facilitate risk stratification of PCa and aid clinical decision-making [20, 5].

Prior studies show that PSMA PET/CT is superior to conventional imaging methods for lymph node metastatic detection, and that the IPSA level is correlated with the PSMA-targeting PET-tracer accumulation in the primary PCa $[21,18]$. However, to our knowledge, no prior studies have employed ${ }^{18} \mathrm{~F}$ PSMA-1007 PET/CT to evaluate the diagnostic performance of tPSA levels to distinguish primary PCa patients with distant metastatic from those without. The present study aims to assess the predictive performance of tPSA level based on ${ }^{18} \mathrm{~F}$-PSMA-1007 PET/CT findings using receiver operating characteristic (ROC) curve. In addition, we aim to evaluate the relation between prediction tPSA level and the semi-quantitative index of maximum Standardized Uptake Value (SUVmax) in the detection of distant metastasis.

\section{Materials And Methods}

\section{Patients}

We performed retrospective analysis for one hundred and one patients with primary PCa confirmed by biopsy or radical prostatectomy between March 2019 and June 2020. All participants included in the data analysis were evaluated by ${ }^{18} \mathrm{~F}-\mathrm{PSMA}-1007$ PET/CT and had total PSA value measured within 4 weeks prior to the ${ }^{18} \mathrm{~F}-\mathrm{PSMA}-1007$ PET/CT imaging. Diagnosis of PCa proven through histological examination served as reference for the PET imaging analyses. Patients were excluded from analysis if they 1) had 
received local or systemic treatment, 2) lacked histological examination proven diagnosis or tPSA value, 3) had incomplete imaging data. This study was approved by local ethics committee (No. 2019LSYZD$\mathrm{J} 1 \mathrm{H}$ ) and was conducted in accordance with the Declaration of Helsinki. Informed consent was obtained from each patient.

\section{${ }^{18}$ F-PSMA-1007 and image acquisition}

All ${ }^{18}$ F-PSMA-1007 PET/CT data was acquired on a PET/CT scanner (Gemini 64TF, Philips, Netherlands) at a single location (The First Affiliated Hospital of Xi'an Jiaotong University). Radiolabeling was performed using a fully automated radiopharmaceutical synthesis device based on a modular concept (MINItrace, GE Healthcare, USA). Over $99 \%$ radiochemical purification yield ${ }^{18} \mathrm{~F}$-PSMA was obtained and examined by both radio-thin layer chromatography (TLC) and high performance liquid chromatography (HPLC) analysis.

Patients received intravenous injection of ${ }^{18} \mathrm{~F}-\mathrm{PSMA}-1007$ (3.7 MBq/kg body weight), and completed PET and CT scans 90 minutes after the injection. Low-dose CT scans from head to the proximal thighs (pitch $0.8 \mathrm{~mm}, 60 \mathrm{~mA}, 140 \mathrm{kV}$ [peak], tube single turn rotation time $1.0 \mathrm{~s}$ and 5-mm slice thickness) for PET attenuation were acquired (pitch $0.8 \mathrm{~mm}$, automatic $\mathrm{mA}, 140 \mathrm{kV}$ [peak] and $512 \times 512$ matrix). Wholebody PET scans were performed in three-dimensional mode (emission time: 90 s per bed position, scanned at a total of 7-10 beds).

\section{Imaging analysis}

All ${ }^{18}$ F-PSMA-1007 PET/CT images were analyzed using Fusion Viewer software in the Extended Brilliance Workstation (EBW, Philips, Netherlands). Two experienced nuclear medicine specialists jointly interpreted all ${ }^{18} \mathrm{~F}$-PSMA-1007 PET/CT scans, and performed comprehensive analysis of available clinical data. Consensuses were achieved through discussion when conclusions between the two specialists were discordant. The SUVmax of the primary PCa was calculated automatically with a manually adapted isocontour threshold centered on lesions with focally increased uptake corresponding to the tumor site verified by TRUS biopsy or radical prostatectomy [18]. The SUVmax values were also calculated for metastases, defined by an uptake higher than the local background and not associated with physiologic uptake per the guideline of the Society of Nuclear Medicine and Molecular Imaging and the European Association of Nuclear Medicine. The identified metastases were also consistent with PCa lesions pathologic tracer accumulation $[22,23,5]$. PET/CT scan findings were classified as (a) primary tumor, and (b) distance metastasis (abdominal lymph nodes, bone and internal organs) (Fig 1).

\section{Statistical analysis}

The Shapiro-Wilk W test was used to test for normal distribution of all continuous variables. Based on data normality, independent two-sample t test and the Mann-Whitney $U$ test were used for group comparison accordingly. Based on PET/CT findings, the participants were divided into non-metastasis and metastasis groups. The diagnostic performance of a prediction tPSA value was evaluated using the 
ROC curve and the area under the curve (AUC). Additionally, a logistic regression model was created with the metastasis results entered as a binary outcome variable. Four independent variables were included: a binary variable of tPSA value greater or lesser than the prediction IPSA value, a binary variable of tPSA value greater or lesser than the reference value of tPSA per the European Association of Nuclear Medicine guideline, and continuous variables of age and SUVmax. Based on the optimal prediction TPSA cutoff value, we divided all patients into two groups (i.e., high-tPSA and low-tPSA). We then divided each group into three subgroups based on their metastasis status (i.e., primary prostate tissue without metastasis, primary prostate tissue with metastasis, and primary PCa metastasis foci). We performed ANOVA analysis to compare the SUVmax among the three subgroups of high-tPSA and low-tPSA participants, respectively. Finally, we performed path analyses to examine the potential mediating role of tPSA level on SUVmax across different metastatic statuses. Specifically, the mediation model included the SUVmax of primary PCa lesions as the predictor, tPSA level as the mediator, and the SUVmax of metastasis foci as the outcome. A significance level of $a=0.05$ (two-tailed) was applied. Statistical analyses were performed using IBM SPSS Statistics version 22 (IBM Corp., Armonk, NY, USA), GraphPad Prism software, version 8.4 (GraphPad Software, Inc., La Jolla, CA, USA) and MedCalc version 19.0 (MedCalc Software Ltd, Belgium).

\section{Results}

Demographic information and clinical characteristics of the participants were summarized in Table 1. A total of 208 lesions were identified using ${ }^{18} \mathrm{~F}$-PSMA-1007 PET/CT in the 101 patients with primary PCa. Among those lesions, 101 were primary prostate tumors, and 107 were distant metastases lesions detected in 63 patients. The median SUVmax of primary PCa (26.00) was significantly higher than that of the metastatic lesions $(16.90 ; P=0.001, F=18.53)$.

With a cutoff value of 29.01 for the prediction tPSA level, the sensitivity and specificity for detecting metastasis were $57.14 \%$ and $73.68 \%$, respectively, with an AUC of $0.656(95 \% \mathrm{Cl}: 0.555-0.748, \mathrm{P}=0.004)$ (Fig 2).

\section{Table 1 Demographic and clinical characteristics of the 101 study participants}




\begin{tabular}{|ll|}
\hline Characteristic & Value \\
\hline Age (years) & $72(50-90)$ \\
Median (range) & \\
\hline Mean \pm SD & $71.39 \pm 8.72$ \\
\hline tPSA (ng/mL) & $24.97(0.17-2139)$ \\
Median (range) & $123.57 \pm 296.50$ \\
Mean \pm SD & \\
\hline Non-metastatic Patients (\%) & $38(37.6 \%)$ \\
\hline Metastatic Patients (\%) & $63(62.4 \%)$ \\
SUVmax & $26.00(5.95-101.89)$ \\
Primary PCa Median (range) & $29.29 \pm 19.52$ \\
Primary PCa Mean \pm SD & $16.90(5.44-150.24)$ \\
Metastatic Lesions Median (range) & $22.32 \pm 20.08$ \\
Metastatic Lesions Mean \pm SD & \\
\hline
\end{tabular}

Note: PCa: prostate cancer, SD: standard deviation. tPSA: total PSA.

To further evaluate the diagnostic strength of tPSA level for metastasis, tPSA level compared to the prediction tPSA $(29.01 \mathrm{ng} / \mathrm{mL})$, tPSA level compared to the reference tPSA $(20 \mathrm{ng} / \mathrm{mL})$, age and SUVmax were entered as independent variables in a logistics regression. The metastasis status was entered as a binary outcome variable. As shown in Figure 3, the logistic regression found the prediction tPSA level $(29.01 \mathrm{ng} / \mathrm{mL})$ to be the only significant predictive factor ( $P=0.008$, OR [odds ratio]: $3.273,95 \% \mathrm{Cl}: 1.384-$ 7.738).

To better identify the diagnostic performance of the prediction tPSA cutoff value of 29.01, we divided PCa patients into six subgroups based on their tPSA level (i.e., tPSA $<29.01$, tPSA $\geq 29.01$ ) and metastasis staging (i.e., primary prostate tissue without metastasis, primary prostate tissue with metastasis, and primary PCa metastasis focis). ANOVA analysis found that SUVmax was significantly different among the high-tPSA ( $\geq 29.01)$ subgroups $(P=0.001)$ but not the low-tPSA subgroups $(P=0.175)$. Post-hoc analysis showed that, among the high-tPSA subgroups, only the primary prostate tissue with metastasis group and the primary PCa metastasis foci group demonstrated significantly different SUVmax level ( $\mathrm{P}<$ 0.001) (Fig 4). In addition, we compared the SUVmax in individuals with the same metastasis status but different levels of tPSA. SUVmax was found to be significantly higher in high-tPSA metastasis focis subgroup than the low-tPSA metastasis focis subgroup $(P=0.034)$ (Fig 5$)$. We also performed the spearman correlation analyses between tPSA and presence or absence of metastases (binary variables), primary PCa SUVmax and presence or absence of metastases (binary variables) separately, the results 
showed both tPSA and SUVmax associated with metastases $(P=0.008$ and $P=0.014$, respectively) (Fig $6)$.

Lastly, to evaluate the mediation role of tPSA level on SUVmax levels, we constructed a path analysis model by including SUVmax of primary PCa lesions as the predictor, TPSA level as the mediator, and SUVmax of metastasis foci as the outcome. A positive correlation was found between the primary PCa SUVmax and metastasis foci SUVmax $(P<0.001)$. tPSA was found to have a partial mediating effect between the SUVmax values of primary PCa and metastases foci $(P<0.05)(F i g 7)$.

\section{Discussion}

In the present study, our primary finding was that a prediction tPSA level of 29.01 has the potential to detect distant metastasis of PCa. The successful use of prediction tPSA level as a biomarker may help reduce unnecessary invasive examination. In addition, our study found a SUVmax difference between primary tumors and metastatic foci in metastatic PCa patients with a high tPSA level (i.e., > 29.01). A significant mediation model further supports the role of tPSA to differentiate the primary tumors from metastatic foci. In sum, our findings provide a reference for the application of tPSA to screen for distant metastasis of $\mathrm{PCa}$, and provide further evidence for the differences in tumor characteristics between the primary PCa and the distant metastasis.

The tPSA screening is increasingly applied to improve the detection rate of primary PCa. However, due to its prostate organ-specificity, the application of tPSA screening to detect distant metastasis remains controversial [9]. Early detection of distant metastasis and accurate staging is clinically crucial, because the patients may lose the opportunity for radical treatment and face poor prognosis once the primary PCa merges with distant metastasis [24]. PSMA PET/CT is a valuable tool in the individualized prognostic grouping and treatment planning of PCa. Prior studies have demonstrated that PSMA PET/CT is more efficient than traditional imaging methods for detecting distant metastases in primary PCa patients [25$27,20]$. In addition, PSMA PET/CT can accurately detect the primary PCa and distant metastasis at the same time, preventing patients from undergoing repeated inspections and invasive biopsy [28].

Prior studies have established the diagnostic efficacy of PSMA PET/CT for primary PCa and its relationship with PSA levels. Specifically, studies have highlighted that the TPSA level was significantly lower in PCa negative individuals than the PCa positive individuals using ${ }^{68} \mathrm{Ga}-\mathrm{PSMA}-11$ PET/CT $[18,19]$. In our study, we demonstrated the diagnostic efficacy of tPSA value to predict PCa distant metastasis using ${ }^{18} \mathrm{~F}-\mathrm{PSMA}-1007$ PET/CT. This may help establish a new TPSA reference for detecting distant metastasis. In a logistic regression model, we demonstrated that the prediction tPSA level of 29.01 has a predictive value superior to that of the commonly used reference of 20.00 when age and SUVmax were controlled.

Furthermore, a valuable assessment tool for distant metastasis of PCa should be able to accurately describe the characteristics of the metastatic lesions and provide precise information for clinical 
decision-making. Previously used tPSA level in the screening process results in a low specificity, leading to limited clinical utility and an overuse of invasive biopsy. Previous studies using a tPSA cutoff value of $30 \mathrm{ng} / \mathrm{ml}$ in groups of men showed diagnos performance for primary PCa ranging from $90 \%$ to $95.7 \%$ [9, 29]. Among those who were not diagnosed with malignant $P C a$ through biopsy, bone scan still detected positive metastases. To understand how tPSA level may help differentiate the primary tumor from the distant metastasis, we grouped the PCa patients based on their prediction IPSA level. When the TPSA is greater than or equal to 29.01, the SUVmax shows statistically significant difference between primary PCa with metastatic and primary PCa metastatic foci. This could be explained by the fact that the inflammation and hyperplasia with the destruction of glandular epithelium lead to the difference in the characteristics of the prostate lesions. Primary PCa can be a heterogeneous multifocal tumor, and may lead to metastatic lesions with varying characteristics [30-35]. In summary, our results suggest that the predictive level of tPSA of 29.01 may ensure a higher detection rate of primary PCa, and can facilitate ${ }^{18}$ F-PSMA-1007 PET/CT SUVmax to discriminate primary PCa from distant metastasis.

Previous studies suggest that the detection rate of distant metastasis increases with primary tumor malignancy and the tPSA level $[36,37]$. Although not the primary purpose of our study, we found that SUVmax as measured by the ${ }^{18}$ F-PSMA-1007 PET/CT was significantly higher in high-tPSA (i.e., $\geq 29.01$ ) than low-tPSA (i.e., <29.01) individuals only in those with metastasis foci but not primary PCa lesions. This finding may further reflect the specificity of the source of distant metastases and prostate characteristics at different tPSA levels. Additionally, we found a positive correlation between the SUVmax of the primary PCa and that of the metastases foci, with a partial mediating effect by tPSA. Our study suggest that the expressions of prostate cell characteristics are correlated between the primary PCa and the distant metastasis; however, this correlation is partially mediated by the tPSA level. Therefore, a combination of tPSA level and ${ }^{18} \mathrm{~F}-\mathrm{PSMA}-1007$ PET/CT examination may better reflect the heterogeneity in the characteristics of the primary PCa tumor and the metastasis.

Our study was limited by the retrospective data collection and a relatively small sample size. Replicating the observed effects in larger cohorts was of interest. Future work in this area should consider combining $\mathrm{PET} / \mathrm{CT}$ imaging findings with pathological results of distant metastatic lesions to improve overall diagnostic accuracy. In addition, although the SUVmax parameters of ${ }^{18} \mathrm{~F}-\mathrm{PSMA}-1007$ PET/CT demonstrated promising utility, it is important to note the potential impacts of human rating errors when interpreting the ${ }^{18} \mathrm{~F}-\mathrm{PSMA}-1007$ PET/CT scans. Benign hyperplasia and inflammation may interfere with the SUVmax measurements of PCa. Methods to improve the accuracy of SUVmax measurement may help increase detection specificity. Lastly, the malignancy degree of the primary PCa was not included in this study. In future studies, the relationship between malignancy degree of primary PCa, distant metastasis, and tPSA levels may be further explored.

\section{Conclusion}


We demonstrated the utility of a new prediction tPSA cutoff value in detecting distant metastasis in patients with primary PCa. Additionally, we provided evidence to support the utility of incorporating ${ }^{18} \mathrm{~F}$ PSMA-1007 PET/CT at least a partial but important contribution to further characterize the metastasis lesions associated with primary PCa.

\section{Declarations}

Funding: This study was supported by the New Medical and Technology of the First Affiliated Hospital of Xi'an Jiaotong University in 2019 (XJYFY-2019J1) and 2020 Clinical Research Project of the First Affiliated Hospital of Xi'an Jiaotong University (XJTU1AF-CRF-2020-008).

Conflicts of interest/Competing interests: Nothing to report.

Ethics approval: All procedures performed in studies involving human participants were in accordance with the ethical standards of the institutional and performed in line with the principles of the Declaration of Helsinki. This study was approved by local ethics committee (No. 2019LSYZD-J1-H).

Consent to participate: Informed consent was obtained from each participant.

Consent for publication: All authors read and approved the final manuscript. All authors agree to the submission and possible publication of the article.

Availability of data and material: The data that support the findings of this study are available from the corresponding author upon reasonable request.

Code availability: Not applicable.

Authors' contributions: All authors contributed to the study conception and design. Material preparation, data collection and analysis were performed by Zhuonan Wang, Anqi Zheng, Weixuan Dong, Xiang Liu, Wang Yuan, Fan Gao. The first draft of the manuscript was written by Zhuonan Wang and all authors commented on previous versions of the manuscript. All authors read and approved the final manuscript.

\section{References}

1. Siegel RL, Miller KD, Jemal A. Cancer Statistics, 2017. CA Cancer J Clin. 2017;67(1):7-30. doi:10.3322/caac.21387.

2. Global Burden of Disease Cancer C, Fitzmaurice C, Allen C, Barber RM, Barregard L, Bhutta ZA et al. Global, Regional, and National Cancer Incidence, Mortality, Years of Life Lost, Years Lived With Disability, and Disability-Adjusted Life-years for 32 Cancer Groups, 1990 to 2015: A Systematic Analysis for the Global Burden of Disease Study. JAMA Oncol. 2017;3(4):524-48. doi:10.1001/jamaoncol.2016.5688. 
3. Cooperberg MR, Broering JM, Carroll PR. Time trends and local variation in primary treatment of localized prostate cancer. J Clin Oncol. 2010;28(7):1117-23. doi:10.1200/JC0.2009.26.0133.

4. Heidenreich A, Bastian PJ, Bellmunt J, Bolla M, Joniau S, van der Kwast T et al. EAU guidelines on prostate cancer. Part II: Treatment of advanced, relapsing, and castration-resistant prostate cancer. Eur Urol. 2014;65(2):467-79. doi:10.1016/j.eururo.2013.11.002.

5. Zhang J, Shao S, Wu P, Liu D, Yang B, Han D et al. Diagnostic performance of (68)Ga-PSMA PET/CT in the detection of prostate cancer prior to initial biopsy: comparison with cancer-predicting nomograms. Eur J Nucl Med Mol Imaging. 2019;46(4):908-20. doi:10.1007/s00259-018-4255-1.

6. Sanda MG, Cadeddu JA, Kirkby E, Chen RC, Crispino T, Fontanarosa J et al. Clinically Localized Prostate Cancer: AUA/ASTRO/SUO Guideline. Part I: Risk Stratification, Shared Decision Making, and Care Options. J Urol. 2018;199(3):683-90. doi:10.1016/j.juro.2017.11.095.

7. Lam TBL, MacLennan S, Willemse PM, Mason MD, Plass K, Shepherd R et al. EAU-EANM-ESTROESUR-SIOG Prostate Cancer Guideline Panel Consensus Statements for Deferred Treatment with Curative Intent for Localised Prostate Cancer from an International Collaborative Study (DETECTIVE Study). Eur Urol. 2019;76(6):790-813. doi:10.1016/j.eururo.2019.09.020.

8. Semjonow A, Brandt B, Oberpenning F, Roth S, Hertle L. Discordance of assay methods creates pitfalls for the interpretation of prostate-specific antigen values. Prostate Suppl. 1996;7:3-16.

9. Gerstenbluth RE, Seftel AD, Hampel N, Oefelein MG, Resnick MI. The accuracy of the increased prostate specific antigen level (greater than or equal to $20 \mathrm{ng} . / \mathrm{ml}$.) in predicting prostate cancer: is biopsy always required? J Urol. 2002;168(5):1990-3. doi:10.1097/01.ju.0000033330.06269.6d.

10. Heesakkers RA, Hovels AM, Jager GJ, van den Bosch HC, Witjes JA, Raat HP et al. MRI with a lymphnode-specific contrast agent as an alternative to CT scan and lymph-node dissection in patients with prostate cancer: a prospective multicohort study. Lancet Oncol. 2008;9(9):850-6. doi:10.1016/S14702045(08)70203-1.

11. Liu C, Liu T, Zhang N, Liu Y, Li N, Du P et al. (68)Ga-PSMA-617 PET/CT: a promising new technique for predicting risk stratification and metastatic risk of prostate cancer patients. Eur J Nucl Med Mol Imaging. 2018;45(11):1852-61. doi:10.1007/s00259-018-4037-9.

12. Sweat SD, Pacelli A, Murphy GP, Bostwick DG. Prostate-specific membrane antigen expression is greatest in prostate adenocarcinoma and lymph node metastases. Urology. 1998;52(4):637-40. doi:10.1016/s0090-4295(98)00278-7.

13. Marchal C, Redondo M, Padilla M, Caballero J, Rodrigo I, Garcia J et al. Expression of prostate specific membrane antigen (PSMA) in prostatic adenocarcinoma and prostatic intraepithelial neoplasia. Histol Histopathol. 2004;19(3):715-8. doi:10.14670/HH-19.715.

14. Giesel FL, Hadaschik B, Cardinale J, Radtke J, Vinsensia M, Lehnert W et al. F-18 labelled PSMA1007: biodistribution, radiation dosimetry and histopathological validation of tumor lesions in prostate cancer patients. Eur J Nucl Med Mol Imaging. 2017;44(4):678-88. doi:10.1007/s00259-0163573-4. 
15. Oh SW, Cheon GJ. Prostate-Specific Membrane Antigen PET Imaging in Prostate Cancer: Opportunities and Challenges. Korean J Radiol. 2018;19(5):819-31. doi:10.3348/kjr.2018.19.5.819.

16. Kesch C, Kratochwil C, Mier W, Kopka K, Giesel FL. (68)Ga or (18)F for Prostate Cancer Imaging? J Nucl Med. 2017;58(5):687-8. doi:10.2967/jnumed.117.190157.

17. Kuten J, Fahoum I, Savin Z, Shamni O, Gitstein G, Hershkovitz D et al. Head-to-Head Comparison of (68)Ga-PSMA-11 with (18)F-PSMA-1007 PET/CT in Staging Prostate Cancer Using Histopathology and Immunohistochemical Analysis as a Reference Standard. J Nucl Med. 2020;61(4):527-32. doi:10.2967/jnumed.119.234187.

18. Uprimny C, Kroiss AS, Decristoforo C, Fritz J, von Guggenberg E, Kendler D et al. (68)Ga-PSMA-11 $\mathrm{PET} / \mathrm{CT}$ in primary staging of prostate cancer: PSA and Gleason score predict the intensity of tracer accumulation in the primary tumour. Eur J Nucl Med Mol Imaging. 2017;44(6):941-9. doi:10.1007/s00259-017-3631-6.

19. Sachpekidis C, Kopka K, Eder M, Hadaschik BA, Freitag MT, Pan L et al. 68Ga-PSMA-11 Dynamic PET/CT Imaging in Primary Prostate Cancer. Clin Nucl Med. 2016;41(11):e473-e9. doi:10.1097/RLU.0000000000001349.

20. Roach PJ, Francis R, Emmett L, Hsiao E, Kneebone A, Hruby G et al. The Impact of (68)Ga-PSMA PET/CT on Management Intent in Prostate Cancer: Results of an Australian Prospective Multicenter Study. J Nucl Med. 2018;59(1):82-8. doi:10.2967/jnumed.117.197160.

21. Obek C, Doganca T, Demirci E, Ocak M, Kural AR, Yildirim A et al. The accuracy of (68)Ga-PSMA PET/CT in primary lymph node staging in high-risk prostate cancer. Eur $\mathrm{J}$ Nucl Med Mol Imaging. 2017;44(11):1806-12. doi:10.1007/s00259-017-3752-y.

22. Rauscher I, Maurer T, Fendler WP, Sommer WH, Schwaiger M, Eiber M. (68)Ga-PSMA ligand PET/CT in patients with prostate cancer: How we review and report. Cancer Imaging. 2016;16(1):14. doi:10.1186/s40644-016-0072-6.

23. Fendler WP, Eiber M, Beheshti M, Bomanji J, Ceci F, Cho $S$ et al. (68)Ga-PSMA PET/CT: Joint EANM and SNMMI procedure guideline for prostate cancer imaging: version 1.0. Eur J Nucl Med Mol Imaging. 2017;44(6):1014-24. doi:10.1007/s00259-017-3670-z.

24. Gandaglia G, Karakiewicz PI, Briganti A, Passoni NM, Schiffmann J, Trudeau V et al. Impact of the Site of Metastases on Survival in Patients with Metastatic Prostate Cancer. Eur Urol. 2015;68(2):32534. doi:10.1016/j.eururo.2014.07.020.

25. Maurer T, Gschwend JE, Rauscher I, Souvatzoglou M, Haller B, Weirich G et al. Diagnostic Efficacy of (68)Gallium-PSMA Positron Emission Tomography Compared to Conventional Imaging for Lymph Node Staging of 130 Consecutive Patients with Intermediate to High Risk Prostate Cancer. J Urol. 2016;195(5):1436-43. doi:10.1016/j.juro.2015.12.025.

26. Pyka T, Okamoto S, Dahlbender M, Tauber R, Retz M, Heck M et al. Comparison of bone scintigraphy and (68)Ga-PSMA PET for skeletal staging in prostate cancer. Eur J Nucl Med Mol Imaging. 2016;43(12):2114-21. doi:10.1007/s00259-016-3435-0. 
27. Sachpekidis C, Baumer P, Kopka K, Hadaschik BA, Hohenfellner M, Kopp-Schneider A et al. (68)GaPSMA PET/CT in the evaluation of bone metastases in prostate cancer. Eur J Nucl Med Mol Imaging. 2018;45(6):904-12. doi:10.1007/s00259-018-3936-0.

28. Mottaghy FM, Heinzel A, Verburg FA. Molecular imaging using PSMA PET/CT versus multiparametric MRI for initial staging of prostate cancer: comparing apples with oranges? Eur J Nucl Med Mol Imaging. 2016;43(8):1397-9. doi:10.1007/s00259-016-3389-2.

29. Heyns CF, Naude AM, Ahmed G, Stopforth HB, Stellmacher GA, Visser AJ. Serum prostate-specific antigen as surrogate for the histological diagnosis of prostate cancer. S Afr Med J. 2001;91(8):685-9.

30. Neal DE, Jr., Clejan S, Sarma D, Moon TD. Prostate specific antigen and prostatitis. I. Effect of prostatitis on serum PSA in the human and nonhuman primate. Prostate. 1992;20(2):105-11. doi:10.1002/pros.2990200205.

31. Moser PL, Brunner A, Horninger W, Bartsch G, Mikuz G. Correlation between inflammatory cells ( $T$ and B lymphocytes, macrophages) in prostate biopsies and elevated PSA levels in a PSA screening population. Urology. 2002;59(1):68-72. doi:10.1016/s0090-4295(01)01493-5.

32. Schatteman PH, Hoekx L, Wyndaele JJ, Jeuris W, Van Marck E. Inflammation in prostate biopsies of men without prostatic malignancy or clinical prostatitis: correlation with total serum PSA and PSA density. Eur Urol. 2000;37(4):404-12. doi:10.1159/000020161.

33. Berglund E, Maaskola J, Schultz N, Friedrich S, Marklund M, Bergenstrahle J et al. Spatial maps of prostate cancer transcriptomes reveal an unexplored landscape of heterogeneity. Nat Commun. 2018;9(1):2419. doi:10.1038/s41467-018-04724-5.

34. Tu SM, Lin SH, Logothetis CJ. Stem-cell origin of metastasis and heterogeneity in solid tumours. Lancet Oncol. 2002;3(8):508-13. doi:10.1016/s1470-2045(02)00820-3.

35. Hupe MC, Philippi C, Roth D, Kumpers C, Ribbat-Idel J, Becker F et al. Expression of Prostate-Specific Membrane Antigen (PSMA) on Biopsies Is an Independent Risk Stratifier of Prostate Cancer Patients at Time of Initial Diagnosis. Front Oncol. 2018;8:623. doi:10.3389/fonc.2018.00623.

36. Simsek DH, Sanli Y, Engin MN, Erdem S, Sanli O. Detection of metastases in newly diagnosed prostate cancer by using 68Ga-PSMA PET/CT and its relationship with modified D'Amico risk classification. Eur J Nucl Med Mol Imaging. 2020. doi:10.1007/s00259-020-04995-5.

37. Hofman MS, Lawrentschuk N, Francis RJ, Tang C, Vela I, Thomas P et al. Prostate-specific membrane antigen PET-CT in patients with high-risk prostate cancer before curative-intent surgery or radiotherapy (proPSMA): a prospective, randomised, multicentre study. Lancet. 2020;395(10231):1208-16. doi:10.1016/S0140-6736(20)30314-7.

\section{Figures}



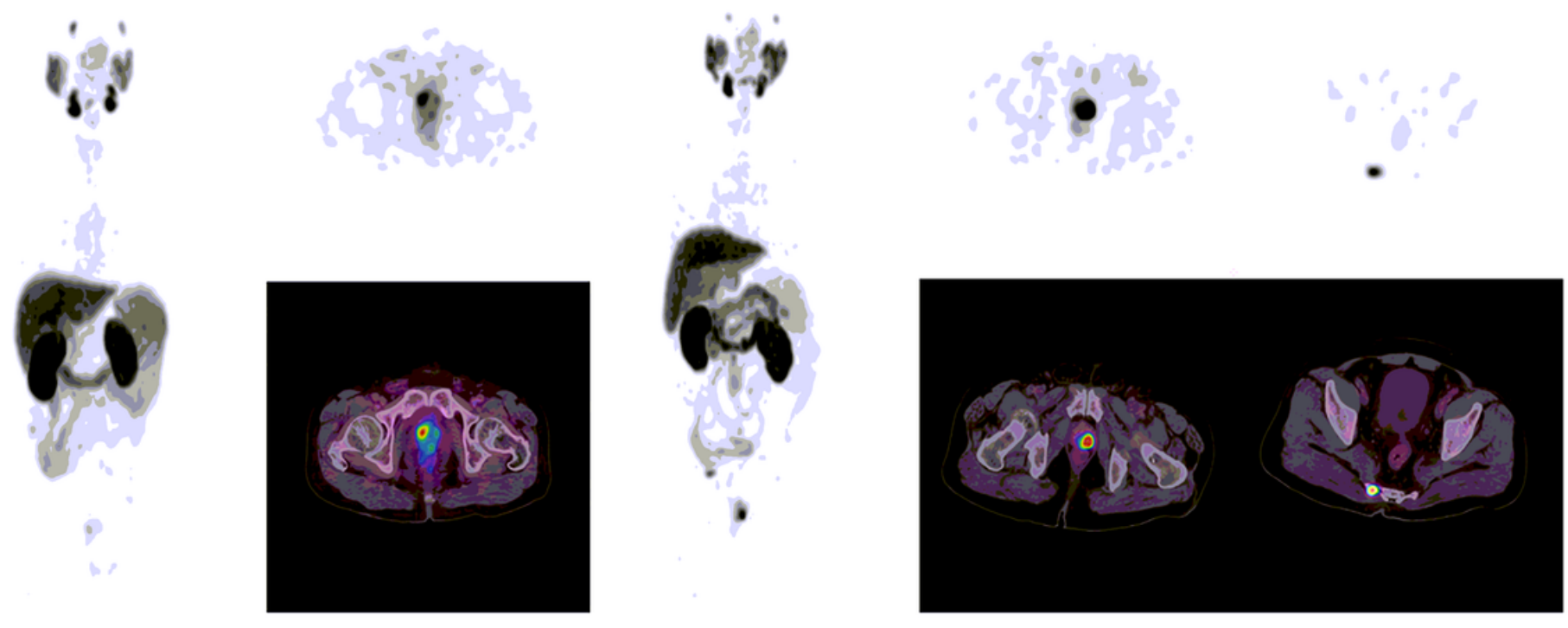

\section{Figure 1}

In the first patient (a), 18F-PSMA-1007 PET/CT results show primary prostate cancer on whole-body maximum intensity projection (MIP) image (left). Axial PET image (right up) and axial fused image (right bottom) show primary tumor in the right prostate cancer lobe. In the second patient (b), 18F-PSMA-1007 PET/CT results show primary prostate cancer and tailbone metastasis on MIP image (left). Axial PET image (middle up) and axial fused image (middle bottom) show primary tumor in the left prostate cancer lobe. Axial PET image (right up) and axial fused image (right bottom) show distant metastasis in tailbone 


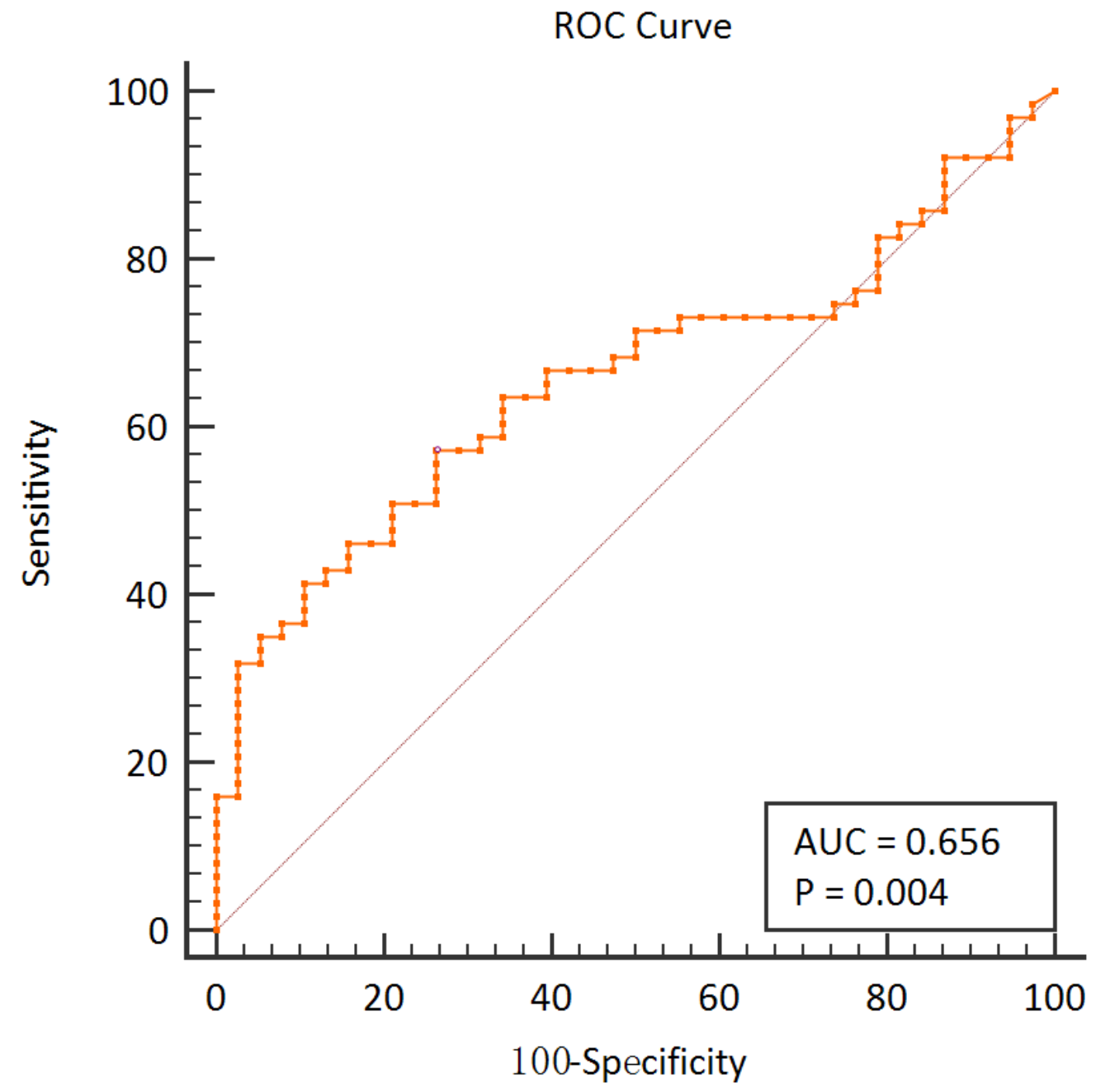

Figure 2

Receiver operating characteristic (ROC) curve of the tPSA level for detecting prostate cancer metastasis with 18F-PSMA-1007 PET/CT

\begin{tabular}{cccc}
\hline Categorical variable & OR & $\mathbf{9 5 \%} \mathrm{Cl}$ & $\mathbf{P}$ \\
\hline SUVmax & 1.02 & $0.997-1.050$ & 0.087 \\
Age & 0.981 & $0.933-1.033$ & 0.473 \\
tPSA = 29.01 & 3.273 & $1.384-7.738$ & 0.008 \\
tPSA = 20 & 2.389 & $1.043-5.472$ & 0.058 \\
\hline
\end{tabular}

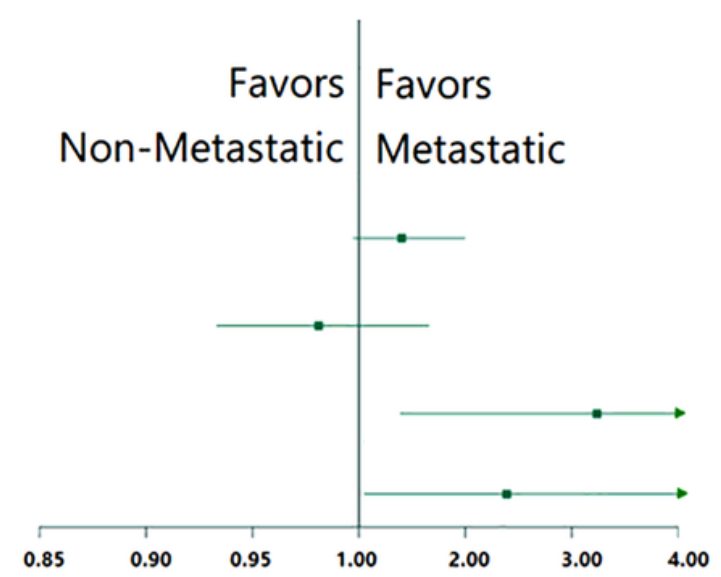

Figure 3 
Logistic analyses of factors predicting prostate cancer metastasis. tPSA: total PSA, OR odds ratio, $\mathrm{Cl}$ confidence interval

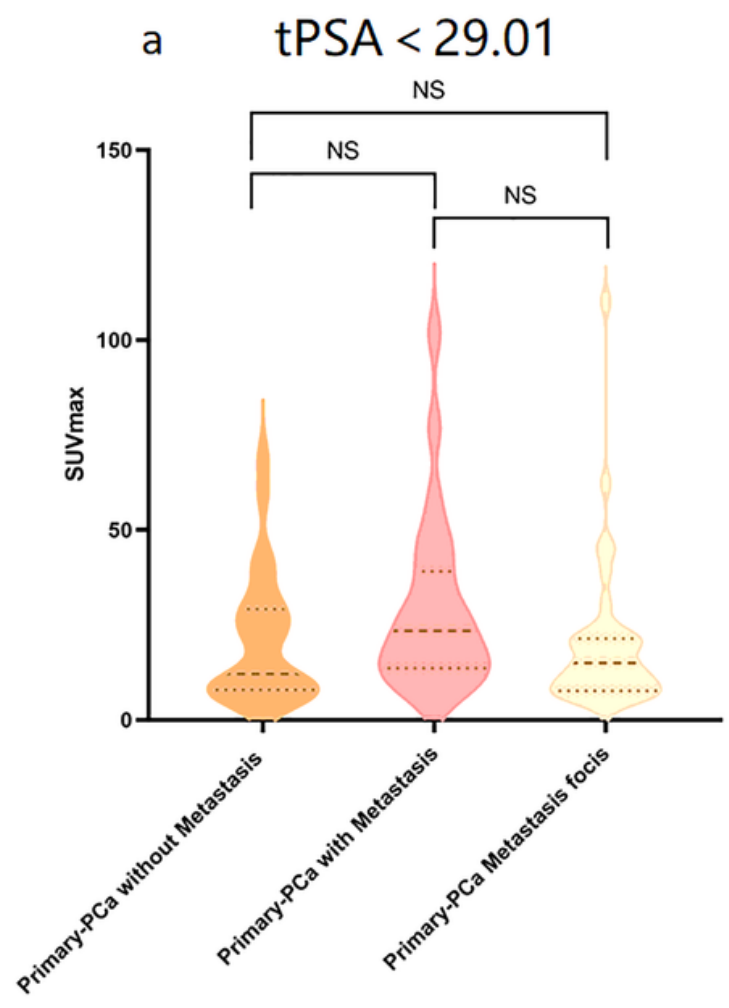

b $\quad$ tPSA $\geq 29.01$

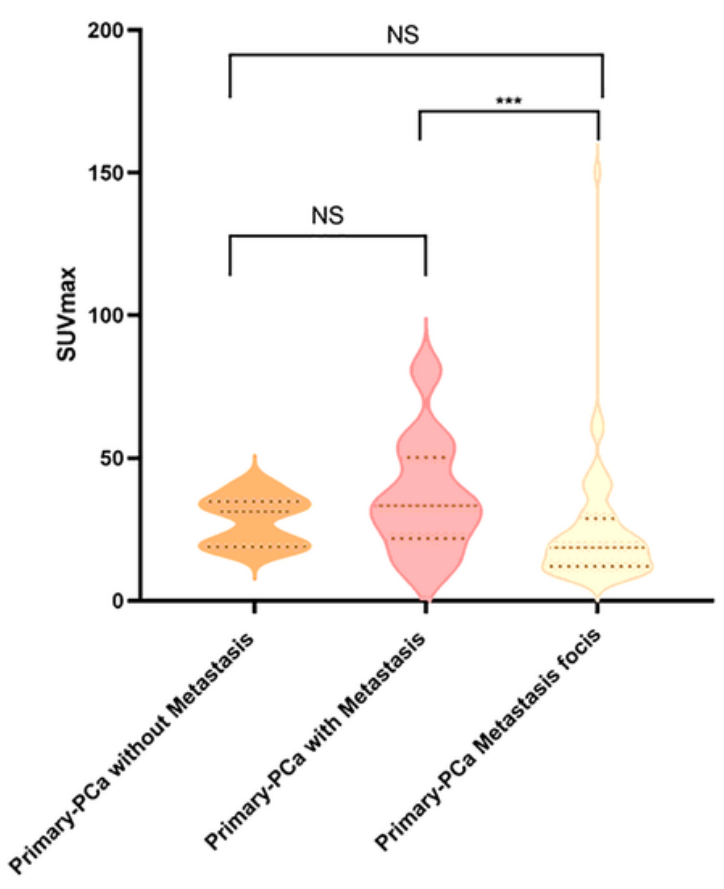

Figure 4

Comparison of 18F-PSMA-1007 SUVmax uptake in primary prostate tissue without metastasis (dark yellow violin box), primary prostate tissue with metastasis (pink violin box) and primary prostate cancer metastasis tissues (light yellow violin box) in tPSA < 29.01 (a) and tPSA $\geq 29.01$ (b). tPSA: total PSA; PCa: prostate cancer 


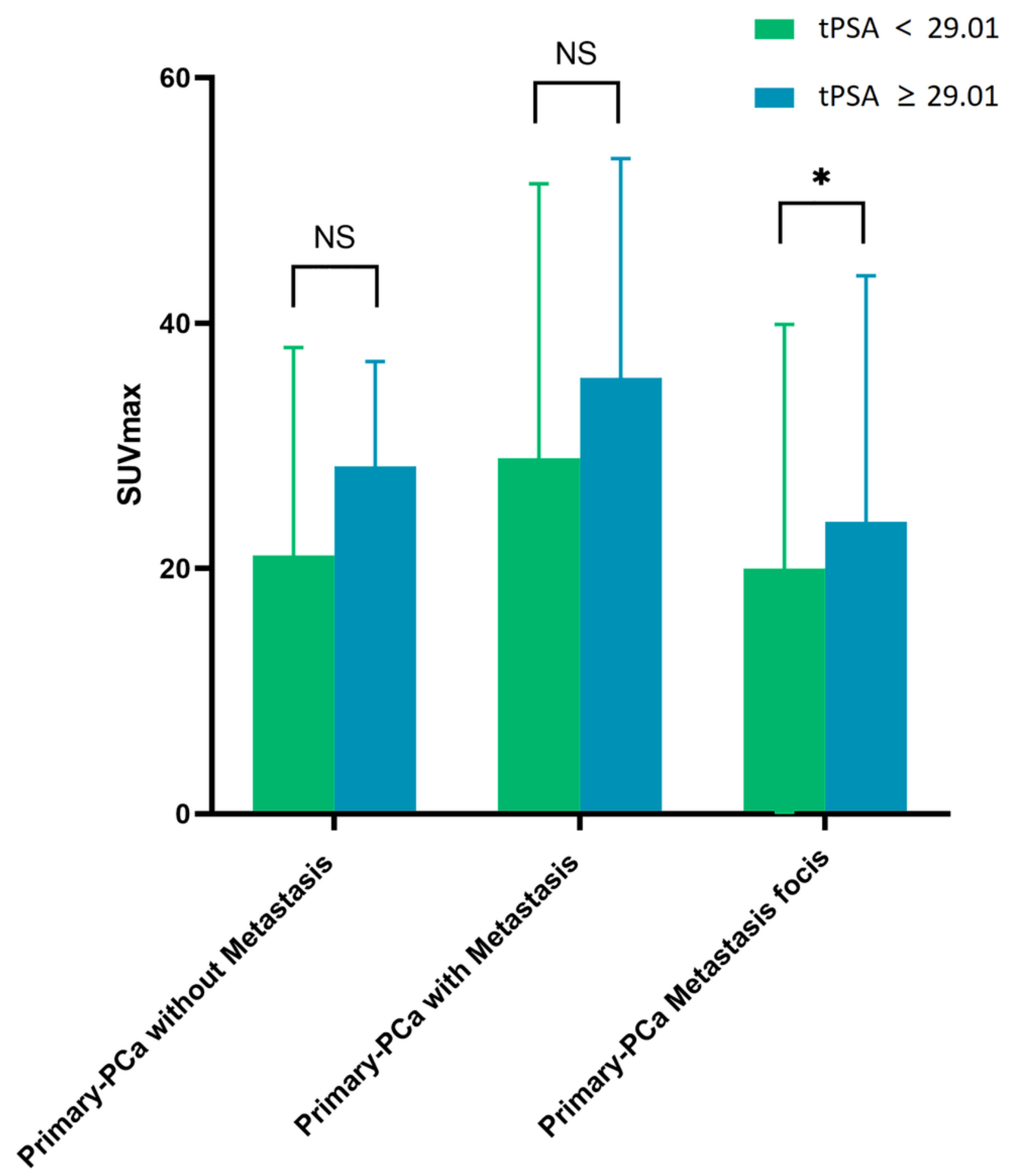

Figure 5

The SUVmax comparison between tPSA $<29.01$ and tPSA $\geq 29.01$ in each sub-group. tPSA: total PSA; PCa: prostate cancer 

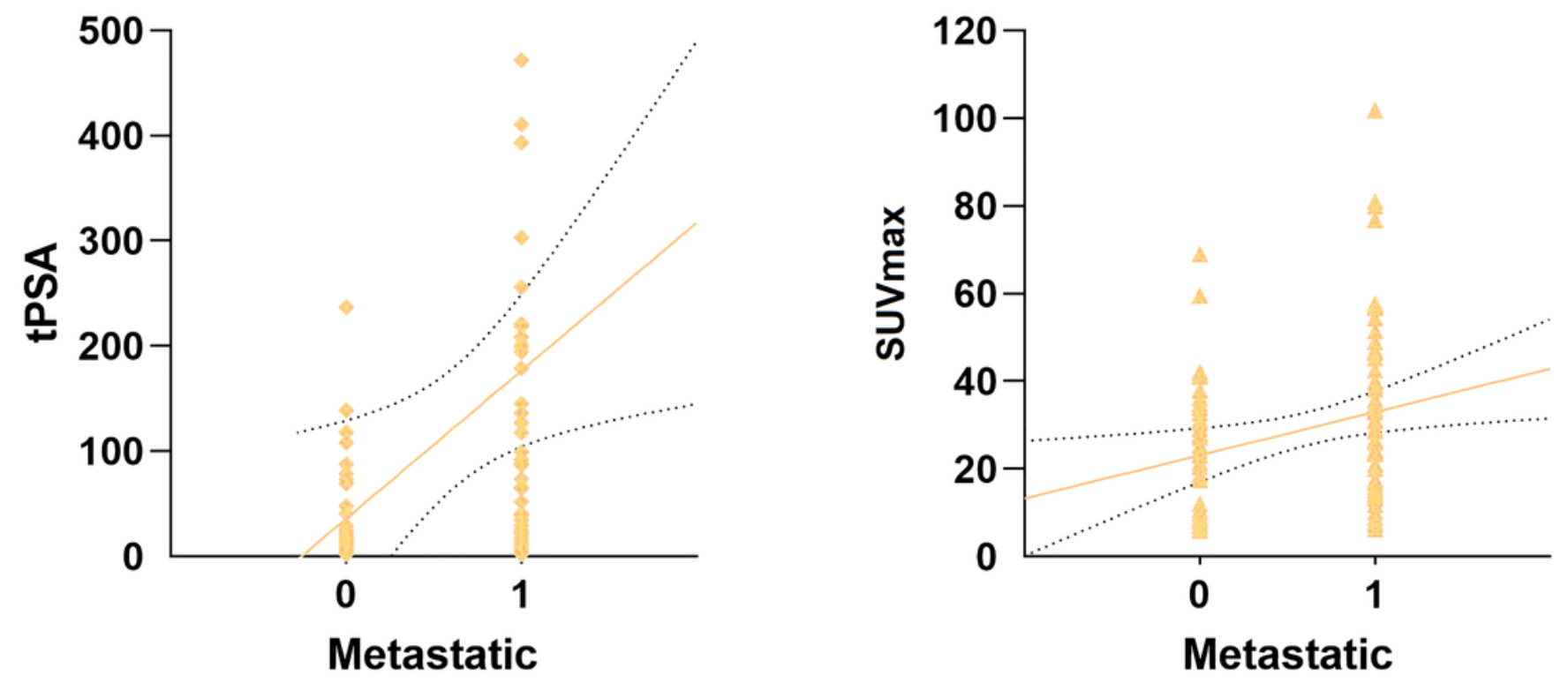

Figure 6

The correlation between primary PCa tPSA and risk of metastatic disease (presence or absence), primary PCa SUVmax and risk of metastatic disease (presence or absence)

\begin{tabular}{|c|c|c|}
\hline $\begin{array}{c}\text { Primary PCa } \\
\text { SUVmax }\end{array}$ & $\mathrm{Y}=0.461 \mathrm{X}+\varepsilon, \mathrm{P}<0.001$ & $\begin{array}{c}\text { Metastatic Focis } \\
\text { SUVmax }\end{array}$ \\
\cline { 2 - 4 }
\end{tabular}

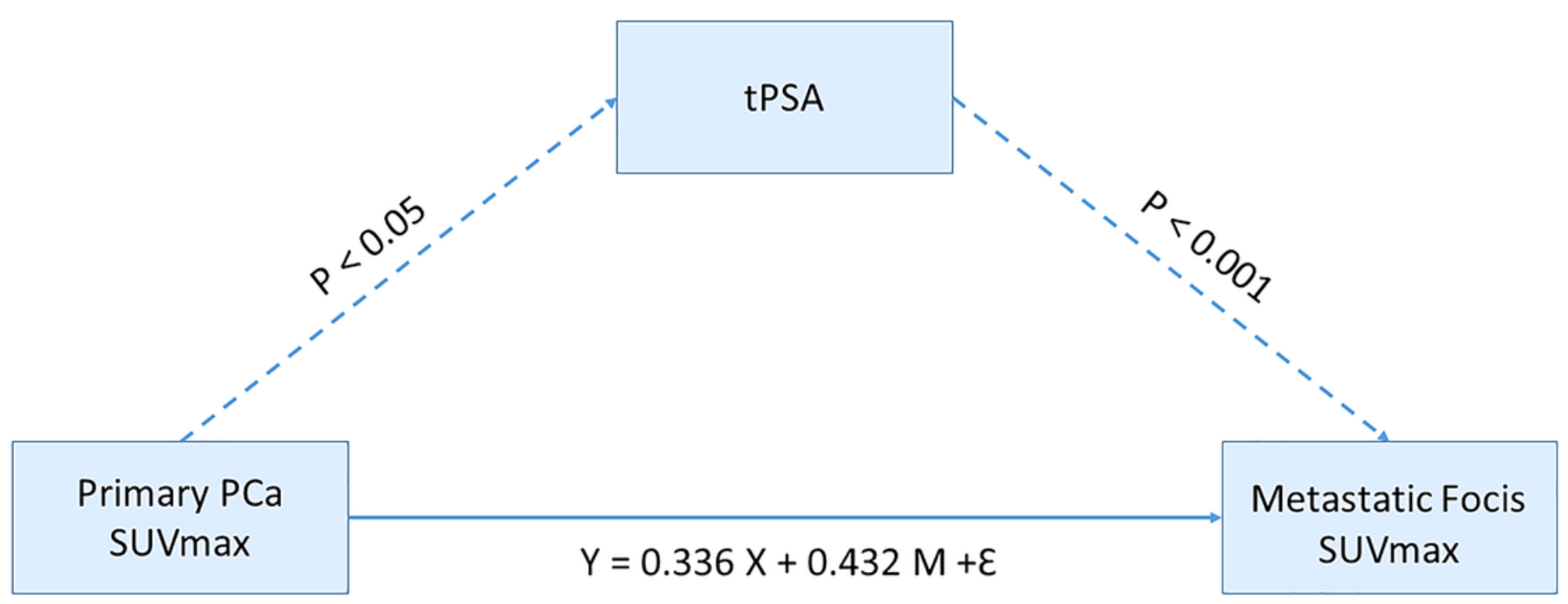

Figure 7 
Mediating effect model between primary prostate cancer SUVmax and Metastatic focis. IPSA: total PSA; PCa: prostate cancer 